\title{
A álgebra das decomposições: mudança estrutural e o desempenho da economia gaúcha no período 1995-2010
}

\author{
Henrique Morrone*
}

Resumo: Este artigo emprega a técnica de decomposições para verificar a contribuição das atividades para o desempenho da economia gaúcha entre 1995 e 2010. Especificamente, duas variáveis são analisadas: a taxa de crescimento da produtividade do trabalho e a relação emprego-população. Essas variáveis servem como medida de performance econômica. A técnica utilizada por Ocampo et al. (2009) foi empregada a fim de verificar a contribuição setorial, bem como a direção da mudança estrutural da economia gaúcha. Essa técnica alternativa permite acessar as fontes de crescimento da economia via um enfoque dinâmico, complementando as análises tradicionais e preenchendo uma lacuna na literatura sobre crescimento regional. Os resultados mostram um padrão de mudança estrutural contrário ao encontrado em economias com crescimento acelerado, indicando que medidas devem ser implementadas a fim de reverter esse processo. Ademais, observou-se que fatores internos aos setores (por exemplo, learning by doing e economias de escala dinâmicas) são mais importantes do que deslocamentos intersetoriais de mão de obra na determinação do crescimento, ratificando a tese Kaldoriana sobre o papel da indústria no crescimento.

Palavras-Chave: Decomposições. Indústria. Mudança estrutural.

\section{The algebra of decomposition: structural change and economic performance in Gaúcha's economy in the period 1995-2010}

\begin{abstract}
This article applies the technique of decomposition in order to determine the contribution of each activity to the performance of the state economy in the period 1995-2010. Specifically, two variables are analyzed: the growth rate of labor productivity and the employment-population ratio. These variables serve as a measure of economic performance. The decomposition technique was employed to estimate sector's contribution, as well as the direction of structural change of the state economy. This technique allows to access alternative sources of growth of the economy via a dynamic approach, complementing traditional analysis and filling a gap in the literature on regional growth. The results show a pattern of structural change contrary to that found in rapidly growing economies, indicating that measures should be implemented to reverse this process. Moreover, it was observed that internal factors to the sectors (e.g., learning by doing and dynamic economies of scale) are more important than intersectoral shifts of labor in determining the growth, confirming the Kaldorian thesis on the role of industry to steam economic growth.
\end{abstract}

Keywords: Decompositions. industry. structural change.

Classificação JEL: O1; O140; R1.

\footnotetext{
* Professor Adjunto da Universidade Federal do Rio Grande do Sul (UFRGS). E-mail: hmorrone@ hotmail.com.
} 


\section{Introdução}

Duas questões centrais em economia dizem respeito às possíveis fontes do crescimento regional e à relação entre a mudança estrutural e o nível de atividade local. Segundo estruturalistas, o processo de desenvolvimento econômico está atrelado a uma mudança estrutural em direção a atividades com economias de escala dinâmicas ${ }^{1}$. Nesse quesito, a indústria de transformação e segmentos do setor de serviços, por apresentarem elevada produtividade e fortes encadeamentos intersetoriais, ganham destaque. Esses setores teriam o potencial para aumentar a produtividade agregada através do efeito transbordamento e das leis de Kaldor. Em contraste, a agricultura teria por função primordial a oferta de alimentos e insumos a um baixo custo a fim de evitar efeitos adversos na competitividade externa e para combater a pobreza (VON ARNIM; RADA, 2011). O papel ativo do setor industrial no que tange à elevação da produtividade é notório. No entanto, esse setor geralmente emprega um volume de mão de obra menor do que o setor de serviços (OCAMPO et al., 2009).

Logo, uma demanda robusta (geradora de emprego) acompanhada da transferência de mão de obra para setores mais produtivos torna-se fundamental para a sustentabilidade do processo de expansão regional.

Nesse contexto, o presente estudo tem por objetivo principal avaliar a contribuição da agricultura, da indústria e dos serviços para o crescimento da economia gaúcha entre 1995 e 2010. Para isso, decompomos duas variáveis: a taxa de crescimento da produtividade do trabalho e a taxa de crescimento da relação emprego/população. Conjuntamente, essas variáves servem como uma proxy do desempenho econômico. Existe uma associação positiva entre crescimento econômico persistente, taxas de expansão da produtividade e uma relação emprego/população crescente (Ocampo et al., 2009). Crescimento econômico persistente ocorre quando há um processo de mudança estrutural em direção a atividades com economias de escala dinâmicas como, por exemplo, o crescimento das economias da Coréia do Sul, Malásia, Cingapura, e Taiwan pós 1950, centrado na indústria e nos serviços. Cabe frisar, que economias de escala dinâmicas são comumente encontradas no setor de manufaturas e serviços high tech. Nessa linha, pode-se comparar o padrão do crescimento da economia com a de outras regiões que apresentem crescimento acelerado.

Ademais, verificar-se-á as fontes do crescimento da produtividade do trabalho estadual. Dois efeitos principais contribuem para o crescimento da produtividade: o efeito

\footnotetext{
${ }^{1}$ Economias de escala dinâmicas são o resultado do aprendizado no processo produtivo e da especialização.
} 
realocação e o efeito intrassetorial. O primeiro seria proveniente do impacto das mudanças de composição do emprego intersetorial na produtividade do trabalho. Tradicionalmente, a migração de mão de obra de um setor de baixa produtividade para um setor mais produtivo seria um exemplo do efeito realocação. Este seria uma peça fundamental nos modelos de economia dual. Já o efeito intrassetorial ${ }^{2}$ estaria relacionado ao aumento da produtividade em setores com mão de obra abundante, ou com economias de escala dinâmicas. Poderemos, assim, detectar as fontes de crescimento e averiguar quais setores seriam os maiores responsáveis pela taxa de crescimento da produtividade agregada.

Para atender aos objetivos propostos, emprega-se o método de decomposições desenvolvido por Ocampo et al.(2009) a fim de analisar a contribuição da agricultura, da indústria e dos serviços na evolução dessas duas variáveis no período 1995-2010. A técnica de decomposição permite averiguar as fontes do crescimento da produtividade através de um enfoque dinâmico. Nessa linha, essa técnica complementa as análises convencionais baseadas nos métodos estrutural-diferencial e insumo-produto. Com relação à fonte dos dados empregados no artigo, utilizaram-se as estatísticas do PIB setorial da Fundação de Economia e Estatística do Rio Grande do Sul (FEE-RS). As estatísticas da população gaúcha também são provenientes da FEE-RS. Ademais, utilizamos os dados referentes ao emprego setorial do Ministério do Trabalho. O estudo inova ao mensurar as contribuições setoriais e as fontes para o crescimento estadual, utilizando um método alternativo de análise. Nesse sentido, o artigo preenche uma lacuna na literatura sobre mudança estrutural e crescimento regional.

O restante do artigo está organizado em quatro seções: a próxima seção apresenta uma breve revisão do papel da agricultura, da indústria e dos serviços no desenvolvimento econômico; a seção 2 apresenta a metodologia; a seção 3 exibe os resultados; e a seção 4 apresenta as conclusões.

\section{O papel da agricultura, da indústria, e dos serviços no desenvolvimento econômico: Uma breve revisão e os Pós-Keynesianos}

$\mathrm{A}_{\text {agricultura }}{ }^{3}$, a indústria e os serviços são setores fundamentais no processo de desenvolvimento econômico. A agricultura possui potencial como atividade indutora da demanda, sendo importante na geração de economias de escala para a indústria doméstica. Esse setor desempenha um papel diferenciado em países em desenvolvimento, onde as divisas

\footnotetext{
${ }^{2}$ Os termos efeito intra-setorial e ganhos de produtividade serão empregados de forma intercambiável neste artigo.

${ }^{3}$ A partir da seção 3, os termos agricultura e agropecuária serão utilizados de forma intercambiável. Isto se dá pela limitação dos dados disponíveis para a economia gaúcha, que são apresentados de forma agregada. No entanto, acreditamos que isso não representa uma limitação à análise dos resultados.
} 
provenientes das exportações criam as condições necessárias à importação de máquinas e equipamentos, tão importantes no processo de industrialização.

Além disso, o setor agrícola pode funcionar às vezes como um reservatório de mão de obra, aumentando o número de empregados em momentos de contração econômica e reduzindo em momentos de expansão (RADA, 2010). Para uma industrialização rápida tornase importante um setor agrícola forte que forneça alimentos de baixo custo, caso contrário o processo de industrialização pode ser abortado (HARRIS; TODARO, 1970; LEWIS, 1954; VON ARNIM; RADA, 2011). Nesse sentido, Lewis (1954) argumenta que desenvolvimento requer aumento da produtividade agrícola. Assim, segundo a literatura econômica, o setor agrícola apresentaria um papel funcional e passivo no processo de desenvolvimento dos países.

Para os estruturalistas, e também para Kaldor, a indústria assume um papel central no desenvolvimento. Para os primeiros, seria apenas através da industrialização que um país atinge o desenvolvimento. Defensores da teoria da indústria nascente, essa corrente do pensamento econômico sustenta que existe uma tendência decrescente nos termos de troca, prejudicando regiões especializadas em produtos primários. Dessa forma, Prebisch (1959) afirma que a industrialização por substituição de importações seria uma forma viável para se alcançar o desenvolvimento.

Prebisch (1959) considera o setor industrial como estratégico para o desenvolvimento e a independência nacional. Para ele, existe uma dualidade entre áreas desenvolvidas, o centro, e as economias em desenvolvimento, a periferia. $\mathrm{O}$ centro apresenta uma base estrutural homogênea, enquanto a heterogeneidade é a regra na periferia. Ademais, o centro produz e exporta bens manufaturados; já a periferia possui uma especialização em produtos primários.

Essa diferença estrutural explica a tendência a relações de troca desfavoráveis na periferia. Isso ocorre devido à especialização em bens de baixa elasticidade-renda da demanda pela periferia, ensejando que a demanda por produtos agrícolas cresça lentamente como um resultado de um aumento da renda no centro. O resultado do aumento da renda periférica se traduz em aumento da demanda por bens manufaturados, aumentando os preços e gerando pressões no balanço de pagamentos. Em contraste, o aumento da renda no centro engendra a redução das importações agrícolas, reduzindo os preços. Como resultado disso, existe um choque positivo no balanço de pagamentos do centro. 
Em síntese, economias em desenvolvimento deveriam se industrializar a fim de se evitar problemas no balanço de pagamentos. Nesse sentido, a industrialização seria necessária, construindo-se a vantagem comparativa em bens manufaturados. Para os estruturalistas, o desenvolvimento econômico é o resultado de uma intensa mudança estrutural em direção à atividade industrial.

Seguindo um caminho alternativo, Kaldor também observou a centralidade da indústria no processo de crescimento. Kaldor evidenciou três fatos estilizados, posteriormente denominados de leis de crescimento (THIRLWALL, 1983), que descreveriam o processo expansionista dos países, bem como sua dinâmica. Sua primeira lei relacionava a taxa de crescimento da produção manufatureira com a taxa de crescimento do Produto Nacional Bruto (PNB). Segundo essa lei, existia uma relação forte e positiva entre as variáveis, que recebia suporte de seu trabalho empírico. Nessa linha, segundo Feijó e Carvalho (2002), dois fatores seriam responsáveis pelo aumento do PIB. O primeiro seria o peso do setor na produção total da economia e o segundo seria a sua taxa de crescimento, sendo o efeito total dado pelo produto de ambos. Como consequência disso, Kaldor considerou o setor manufatureiro como indutor do processo de crescimento econômico.

Em sua segunda lei, também conhecida como lei de Kaldor-Verdoorn, foi estabelecida uma relação positiva entre a taxa de crescimento da produtividade do trabalho da indústria com a taxa de crescimento de sua produção. A demanda estimula o aumento da produção, gerando o aumento de produtividade em setores com retornos crescentes de escala. Desse modo, o crescimento da produtividade seria endógeno, determinado pelo aumento do produto.

Por fim, sua terceira lei estabelecia uma relação positiva entre a taxa de crescimento da produtividade de toda a economia com as variáveis emprego industrial e produção industrial. De acordo com essa lei, existiria também uma relação negativa entre a variável produtividade do trabalho agregada e a variável taxa de crescimento do emprego não industrial.

A explicação teórica para a referida lei estaria no fato de que quanto maior o crescimento da produção do setor de manufaturas, maior será a transferência de trabalhadores dos demais setores, menos produtivos, em direção ao primeiro. Dessa forma, a transferência de trabalhadores dos setores menos produtivos para a indústria engendrará a expansão da produtividade do setor manufatureiro, bem como dos demais. Nessa lei parte-se da hipótese de que a mão de obra empregada nos demais setores é redundante. Assim, a migração para a 
indústria provocaria o crescimento da produtividade tanto na agricultura, quanto no setor de serviços.

Da exposição das três leis, verifica-se a existência de uma consistência interna entre elas. A taxa de crescimento do produto industrial estimula o aumento da produtividade industrial (dado pela segunda lei), que, por sua vez, devido à migração da mão de obra de outros setores para a indústria, gera o aumento da produtividade do trabalho não industrial, bem como de toda a economia (MAMGAIN, 1999).

Dessa forma, o processo de causação circular cumulativa pode ser sintetizado como sugere Stafford (1989): o crescimento da demanda gera maior produção, estimulando maior produtividade, que afeta positivamente a demanda. Esse ciclo autorreforçador pode ser tanto expansivo quanto recessivo. O crescimento lento da demanda provoca efeitos danosos na produção, que, por sua vez, afeta negativamente a produtividade, causando a redução da demanda.

Em síntese, Kaldor (1975) sustenta que a demanda doméstica e externa por manufaturas determinaria a taxa de crescimento da produtividade dos países. Nesse cenário, o setor industrial seria especial por sua capacidade de estimular um crescimento sustentável.

O setor de serviços recentemente tem sido apontado como um setor com potencial para impulsionar o crescimento. Dasgupta e Singh (2005) sugerem que o setor de serviços pode tornar-se uma atividade pujante, alavancadora de um processo expansionista robusto.

Existem duas interpretações sobre o papel dos serviços no desenvolvimento. Na primeira, o setor de serviços é visto como um complemento da manufatura, sendo subordinado ao desenvolvimento manufatureiro. Kaldor (1968) e Chang (2011) sugerem a existência dessa dependência dos serviços em relação à manufatura.

Em contraste, a segunda vertente afirma que os serviços podem liderar o processo de desenvolvimento de uma economia, servindo como um substituto à manufatura (DASGUPTA; SINGH, 2005). Os serviços poderiam liderar o crescimento das economias, pois eles são cada vez mais tradables, apresentando economias de escala dinâmicas. Nesse contexto, a experiência de crescimento indiana suportaria o argumento em favor dos serviços.

Em resumo, as atividades de serviços poderiam desempenhar um papel ativo ou passivo no processo de crescimento. Torna-se importante diferenciar o setor de serviços high tech, altamente produtivo, do setor serviços de baixa produtividade, predominante em países de renda média, a fim de compreender suas funções no aumento do nível de atividade econômica. 


\section{Procedimentos metodológicos}

As séries do emprego setorial, proveniente do Ministério do Trabalho, do valor adicionado, da população e do valor adicionado setorial foram empregadas nos cálculos das decomposições, no período 1995-2010.Os valores referentes ao valor adicionado setorial (agricultura, indústria e serviços) e a população foram extraídos da Fundação de Economia e Estatística do Rio Grande do Sul (FEE-RS).

Para a avaliação dos resultados, dividimos os dados em dois períodos - 1995 a 2002 e 2003 a 2010. Essa separação decorre da mudança de rumo da economia brasileira, que a partir de meados de 2003 passa a empregar um modelo de crescimento diferenciado, baseado na distribuição de renda e expansão do crédito. Essa nova fase da economia nacional impactou a economia gaúcha, sendo importante comparar os dois períodos. Ademais, a técnica de decomposições exige a comparação de períodos distintos a fim de averiguar a direção da mudança estrutural.

Contudo, os dados da economia gaúcha apresentam duas limitações. Primeiro, os dados para a produtividade foram estimados com base no número de empregados e não no número de horas trabalhadas. Isto ocorre devido à limitação nas estatísticas disponíveis para as horas trabalhadas na produção. Segundo, os valores adicionados de alguns serviços são estimados indiretamente nas contas nacionais, o que exige cautela na interpretação dos resultados.

Nesta seção, apresentamos o método de decomposição empregado para avaliar a contribuição de cada setor (agricultura, indústria, e serviços) na taxa de crescimento da produtividade do trabalho e na relação emprego-população no Rio Grande do Sul. A seção é fortemente influenciada por Divisia (1926), Ocampo et al. (2009), e Roncolato e Kucera (2013).

A produtividade do trabalho agregada da economia pode ser expressa como a razão entre a soma do valor adicionado das atividades e o trabalho empregado para sua efetivação, $\psi=\sum \frac{X^{\hat{1}}}{L^{i}}$. O sobrescrito "i" indica o setor da economia. De forma análoga, calcula-se a taxa de crescimento do produto, $\hat{X}$. Cabe referir, que as taxas de crescimento devem ser calculadas em tempo discreto e infinitesimal. Por exemplo, a taxa de crescimento do emprego, $\hat{L}$, seria expressa da seguinte forma: $\hat{L}=\left(L_{1}-L_{0}\right) / L_{0}$, com os subscritos 0 e 1 representando 
pontos no tempo. Seguindo Ocampo et al. (2009) e após algumas manipulações algébricas ${ }^{4}$, a equação da taxa de crescimento da produtividade setorial pode ser representada da seguinte forma:

$$
\hat{\psi}^{i}=\left(1+\hat{L}^{i}\right)^{-1}\left(\hat{X}^{i}-\hat{L}^{i}\right)
$$

Sendo $\left(1+\hat{L}^{i}\right)^{-1}$ um termo de interação, de pouca importância na explicação do crescimento da produtividade.

Processo semelhante pode ser empregado para a obtenção da taxa de crescimento da produtividade agregada. A taxa de crescimento da produtividade agregada ${ }^{5}$ pode ser escrita como segue:

$$
\hat{\psi}=\sum_{i}\left[\chi_{0}^{i}\left(\hat{X}^{i}-\hat{L}^{i}\right)+\left(\chi_{0}^{i}-\left(\psi_{1}^{i} \beta_{0}^{i}\right) \kappa_{0}^{i}\right) \hat{L}^{i}\right]
$$

Onde $\hat{\psi}$ representa a taxa de crescimento da produtividade do trabalho; $\chi$, a relação produto setorial-produto total; $\hat{X}^{i}$, a taxa de crescimento do valor adicionado do setor $\mathrm{i}$; $\hat{L}^{i}$, a taxa de crescimento do emprego do setor i; $\beta$ a relação emprego-produto (inverso da produtividade); e $\kappa$, a parcela emprego setorial/emprego total. A expressão $\left(\psi_{1}^{i} \beta_{0}^{i}\right)$ é conhecida na literatura como efeito interação.

Além do termo de interação, $\left(\psi_{1}^{i} \beta_{0}^{i}\right)$, que assume um papel secundário, a produtividade agregada pode ser decomposta em duas partes principais: o efeito intra-setorial

\footnotetext{
${ }^{4}$ Matematicamente, pode-se chegar ao resultado da Equação (1) com os seguintes passos. Iniciando-se com a expressão $\hat{\psi}=\frac{X_{1}-X_{0}}{X_{0}}$, pode-se proceder a a adição e subtração indicada, $X_{1} / L_{0}-X_{1} / L_{0}$, e provar que o resultado será idêntico à taxa de crescimento da produtividade, $\hat{\psi}$. A prova matemática está disposta a seguir: 
(ganhos de produtividade) e o efeito realocação. De um modo geral, o efeito intra-setorial e o efeito realocação explicam quase que totalmente a produtividade, sendo preciso em média em até três casas decimais (Ocampo et al., 2009).

O primeiro termo, $\chi_{0}^{i}\left(\hat{X}^{i}-\hat{L}^{i}\right)$, é conhecido na literatura como efeito intra-setorial (ou ganhos de produtividade). Ele é uma média das taxas de crescimento da produtividade setorial ponderada pelo peso dessa atividade no valor adicionado total. Pode-se defini-lo como a variação da produtividade decorrente de fatores internos aos setores. Por exemplo, as economias de escala dinâmicas da indústria, bem como a mão de obra abundante na agricultura seriam duas fontes para o crescimento da produtividade. A primeira faz com que cada trabalhor, através do aumento da escala de produção e do ganho de aprendizado, fique mais produtivo, enquanto a segunda garante o crescimento da produtividade agrícola.

Segundo Kaldor, as economias de escala dinâmicas seriam fundamentais para a indústria. Essas derivam do progresso técnico, das economias externas, da divisão do trabalho e do learning by doing (Feijó e Carvalho, 2002). As economias de escala dinâmicas são o resultado, por exemplo, dos ganhos de aprendizado dos trabalhadores, que se tornam mais eficientes e aptos a executar inovações no processo produtivo (Oliveira, 2002).

A segunda expressão, $\left(\chi_{0}^{i}-\kappa_{0}^{i}\right) \hat{L}^{i}$, representa o efeito realocação, sendo definido como a variação na produtividade derivada da mudança na composição do emprego intersetorial. Caso $\chi_{0}^{i}$ seja maior que $\kappa_{0}^{i}$, o setor apresentará uma parcela no produto total maior que a razão emprego-emprego total, indicando que possui uma produtividade relativamente acima da média da economia (Ocampo et al., 2009). Desse modo, o crescimento do emprego nesse setor resultaria num aumento da produtividade do trabalho da economia. A migração de mão de obra da agricultura, setor com baixa produtividade, à indústria, setor de alta produtividade, implicaria no aumento da produtividade agregada, sendo um exemplo de efeito realocação.

O efeito realocação será positivo quando houver crescimento do emprego em setores com produtividade acima da média da economia (Roncolato e Kucera, 2013), ou em setores de baixa produtividade em que o emprego diminua. Nesse caso, a transferência de trabalhadores da agricultura, um setor de baixa produtividade em alguns países, para a indústria, detentora de alta produtividade, engendrará um crescimento na produtividade média da economia. Com uma elevada relação capital-trabalho e acesso à capital, a indústria pode 
tornar cada trabalhador transferido mais produtivo. Nota-se que o crescimento da produtividade agrícola ocorre de forma passiva.

A taxa de crescimento da produtividade agregada será, portanto, a adição desses dois efeitos para todos os setores da economia ${ }^{6}$. Nesse sentido, quanto mais hetoregênea a estrutura produtiva de um país, maior será o potencial para o aumento da produtividade através da migração de mão de obra, ou seja, maior será o efeito realocação (Roncolato e Kucera, 2013).

Mudando o foco de análise para a segunda variável (a relação emprego-população), cabe frisar que um setor criará emprego se sua taxa de crescimento do produto per capita for superior a taxa de crescimento da produtividade ${ }^{7}$. Pode-se decompor a relação empregopopulação, da seguinte forma:

$$
\rho=L_{0} / P_{0}=\sum_{i}\left(L_{0}^{i} / X_{0}^{i}\right)\left(X_{0}^{i} / P_{0}\right)
$$

Sendo $\rho$ a relação emprego-população, $L_{0}$ o emprego, $X_{0}$ o valor adicionado, e $P_{0}$ a população. Com um pouco de algebra ${ }^{8}$ e usando as variáveis emprego-produto $\left(\varepsilon_{0}^{i}=L_{0}^{I} / X_{0}^{i}\right)$ e produto per capita $\left(\mu_{0}^{i}=X_{0}^{i} / P_{0}\right)$, obtemos a seguinte expressão para a taxa de crescimento da relação emprego-população:

$$
\hat{\rho}=\sum_{i} \kappa_{0}^{i}\left[\hat{\mu}^{i}-\psi^{i}\left(1+\hat{\mu}^{i}\right)\left(1+\hat{L}^{i}\right)\left(1+\hat{X}^{i}\right)^{-1}\right] .
$$

O termo multiplicando a taxa de crescimento da produtividade, $\psi$, é o nosso conhecido efeito interação, sendo o mesmo pouco expressivo, podendo-se desconsiderá-lo da análise. Logo, a taxa de crescimento da relação emprego-trabalho pode ser adicionalmente simplificada, chegando-se em sua expressão final.

$$
\hat{\rho}=\sum_{i} \kappa_{0}^{i}\left(\hat{\mu}^{i}-\psi^{i}\right)
$$

Assim, a taxa de crescimento da parcela emprego-população pode ser definida como a média ponderada pela relação emprego setorial/emprego total da diferença entre as taxas de

\footnotetext{
${ }^{6}$ A magnitude do efeito intra-setorial e do efeito realocação pode ser sensível ao nível de desagregação dos dados brutos. A tendência é de que quanto mais desagregado maior será o impacto do efeito realocação na evolução da taxa de crescimento da produtividade (Roncolato e Kucera, 2013).

${ }^{7}$ A decomposição é eficaz somente se a participação da força de trabalho for estável (Pasinetti, 1981; Ocampo et al., 2009)

${ }^{8}$ Para mais detalhes, ver Ocampo et al. (2009).
} 
crescimento do produto per capita e da produtividade. Setores que apresentem uma parcela emprego setorial/emprego total elevada contribuirão mais na geração de emprego. Setores maduros apresentarão uma taxa de crescimento da produtividade superior a taxa de crescimento do produto per capita, sendo o oposto válido para setores dinâmicos (Ocampo et al., 2009).

Contudo, a abordagem de decomposições apresenta um inconveniente. Apesar de a abordagem permitir comparar as características estruturais de regiões com baixo e acelerado crescimento, ela é incapaz de acessar o efeito de estruturas alternativas sobre o crescimento, impedindo a execução de avaliações contra factuais (RONCOLATO; KUCERA, 2013).

No entanto, a técnica de decomposição desenvolvida por Ocampo et al. (2009) permite avaliar as fontes do crescimento da produtividade através de um enfoque dinâmico. Nessa linha, essa técnica complementa as análises convencionais baseadas nos métodos estruturaldiferencial e insumo-produto ${ }^{9}$. Assim, o procedimento adotado no presente artigo é adequado para examinar-se a evolução da produtivida do trabalho estadual e da razão empregopopulação.

\section{Resultados}

Nesta seção, examinam-se os resultados da decomposição setorial de três variáveischave na mensuração do desempenho da economia gaúcha: a taxa de crescimento do produto, a taxa de crescimento da produtividade e a taxa de crescimento da relação empregopopulação. Além disso, avaliamos as fontes de crescimento setorial e procedemos a análise dos subsetores da indústria.

\subsection{Mudança estrutural e performance da economia gaúcha}

A Tabela 1 apresenta a parcela dos setores no valor adicionado total em dois períodos: 1995-2002 e 2003-2010. Conforme indicado anteriormente, essa divisão dos períodos decorre das mudanças de rumo da política econômica nacional (baseada na distribuição de renda e expansão do crédito a partir de 2003) e seus impactos na economia gaúcha. No primeiro período, a agropecuária representou em média 9,67\% do valor adicionado total. A indústria e o setor de serviços exibiram uma parcela de aproximadamente 29,91\% e 60,42\%,

\footnotetext{
${ }^{9}$ Para uma revisão dos métodos estrutural-diferencial e de insumo-produto, ver respectivamente Souza (2009) e Bêrni (2006). O primeiro apresenta como limitação um viés na avaliação de longos períodos, ao passo que o segundo baseia-se em matrizes de insumo-produto defasadas no tempo. Essas duas limitações são evitadas pelo uso do método de decomposições no presente artigo.
} 
respectivamente. $\mathrm{O}$ peso de cada setor no valor adicionado total é uma medida da contribuição setorial para a geração do produto.

Comparando-se esses resultados com os encontrados no segundo período, constatamos que houve uma mudança estrutural relativamente modesta. Ocorreu uma redução da participação da indústria (aproximadamente 5,6\%) acompanhada do aumento de participação dos serviços e da agropecuária.

Tabela 1: Participação média dos setores no valor adicionado gaúcho (\%)

\begin{tabular}{lcc}
\hline & \multicolumn{2}{c}{ Períodos } \\
\cline { 2 - 3 } Setores & $1995-2002$ & $2003-2010$ \\
\hline Agropecuária & 9,67 & 11,21 \\
Indústria & 29,91 & 28,29 \\
Serviços & 60,42 & 60,50 \\
\hline
\end{tabular}

Fonte: Elaboração própria.

Essa análise pode ser complementada por um estudo da dinâmica de crescimento através da técnica de decomposições. O valor adicionado total é composto pela soma dos produtos setoriais, $X=\sum_{i=1}^{n} X_{i}$. A diferenciação dessa equação com relação ao tempo implica que a taxa de crescimento do produto torna-se a soma das médias ponderadas das taxas de crescimento do produto setorial, $\hat{X}=\sum_{i=1}^{n} \chi_{i} \hat{X}_{i}$.

O Gráfico 1 mostra a contribuição setorial para o crescimento do produto nos períodos 1995-2002 e 2003-2010. No primeiro, a taxa de crescimento industrial representava em média $28,15 \%$ da taxa de crescimento do produto total da economia, caindo essa participação para $13,43 \%$, uma queda de aproximadamente 53\%. Já a agropecuária aumenta sua contribuição para o crescimento em cerca de $117 \%$. Esta última foi beneficiada pelo ciclo de valorização das commodities (Bender Filho, 2013). O setor de serviços seria um caso intermediário, havendo crescimento em sua contribuição, porém, num montante inferior ao da agropecuária. Isto é um indicativo de uma mudança estrutural reversa da economia estadual.

De acordo com o Gráfico 1, uma conclusão crucial merece destaque: a queda da contribuição industrial para o crescimento do valor adicionado, seguida do aumento da contribuição dos setores agropecuário e de serviços. Em outras palavras, os resultados da decomposição revelam que o crescimento do produto total está sendo determinado majoritariamente pelos serviços e pela agropecuária. 


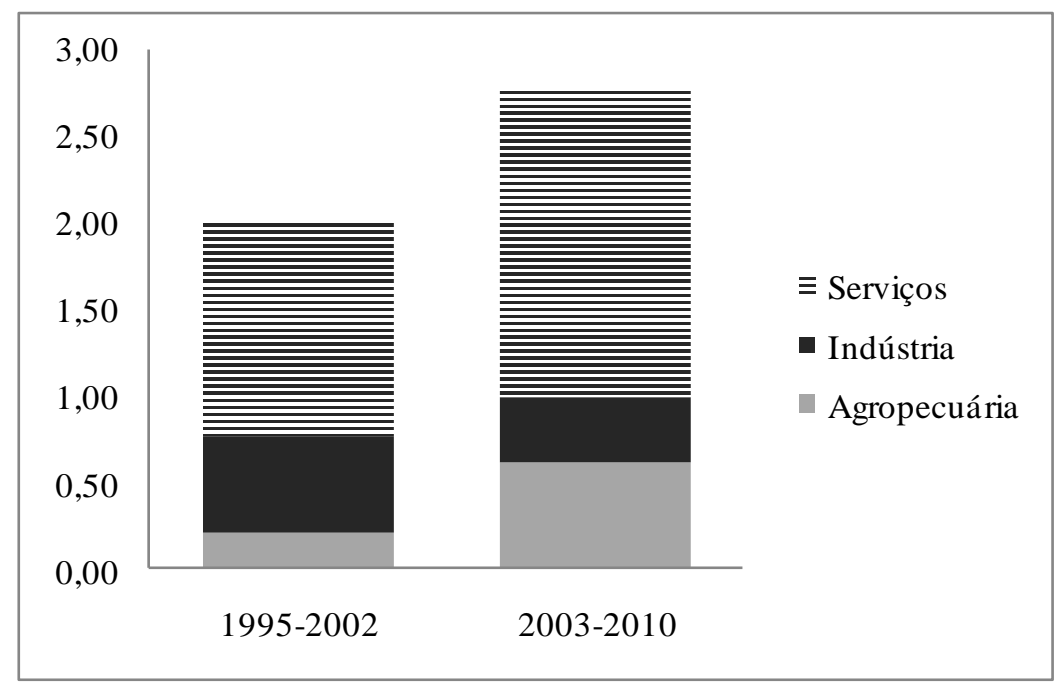

Gráfico 1: Contribuição setorial para o crescimento do produto da economia gaúcha (\%) Fonte: Elaboração própria.

A Tabela 2 apresenta à decomposição da variância da taxa de crescimento do produto estadual. Os resultados revelam que a taxa de crescimento do produto industrial explicava $69,5 \%$ da variância do crescimento do valor adicionado total no período 1995-2002. No segundo período, a indústria passa a explicar apenas 33,9\% da variância total. Deste modo, constata-se um aumento da importância dos setores agropecuário e do de serviços na explicação da variância da taxa de crescimento do produto regional.

Tabela 2: Coeficiente de covariância entre o crescimento do produto setorial e da taxa de crescimento do valor adicionado gaúcho $(\%)$

\begin{tabular}{lcc}
\hline & \multicolumn{2}{c}{ Períodos } \\
\cline { 2 - 3 } Setores & $1995-2002$ & $2003-2010$ \\
\hline Agropecuária & 6,114 & 33,933 \\
Indústria & 69,545 & 33,931 \\
Serviços & 24,341 & 32,136 \\
\hline Fonte: Elaboração própria. &
\end{tabular}

Ademais, o Gráfico 2 exibi os resultados para a decomposição da taxa de crescimento da produtividade. Analisando-se o primeiro período (1995-2002), observamos que o crescimento da produtividade da agropecuária impede uma queda mais acentuada da produtividade agregada. Nesse período, a relativa estagnação da produtividade industrial não foi determinante do resultado agregado. Com efeito, foi o setor de serviços que determinou os resultados negativos. A performance agregada $(-0,41 \%)$ está num patamar muito inferior a observada em economias com crescimento rápido, como, por exemplo, as economias 
asiáticas ${ }^{10}$. Desse modo, os resultados setoriais culminaram numa situação de retração da produtividade.

No segundo período (2003-2010) há um aprofundamento das tendências observadas previamente. Existe um aumento da contribuição do setor agropecuário para o crescimento da produtividade agregada, confirmando-se a importância do mesmo para a economia gaúcha. Contudo, essa contribuição novamente é insuficiente para compensar os resultados negativos da indústria e dos serviços.

O setor industrial apresentou uma queda média de $0,95 \%$ ao ano. O setor de serviços exibiu resultados ainda piores. Sua taxa de crescimento média anual foi de $-1,23 \%$. Esses dois setores determinaram os resultados negativos do crescimento da produtividade agregada ($1,52 \%)$.

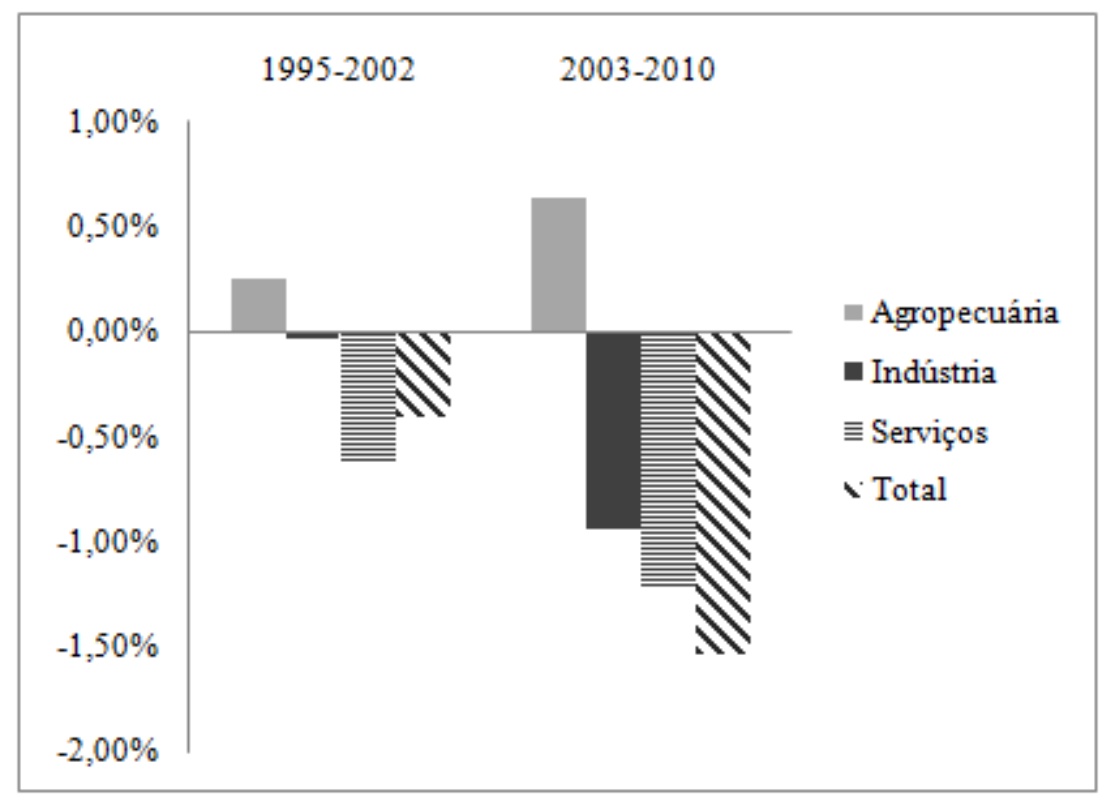

Gráfico 2: Crescimento da produtividade e contribuições setoriais, (\%) Fonte: Elaboração própria.

A análise das decomposições revela uma associação negativa entre a mudança estrutural e o desempenho da economia gaúcha. A despeito da perda do peso da indústria no produto final da economia, esse setor continua sendo importante na explicação do crescimento da produtividade agregada. Ademais, os resultados indicam que o setor de serviços é formado primordialmente por atividades de baixa produtividade. $\mathrm{O}$ aprofundamente dessa tendência representa uma ameaça para a sustentabilidade do processo de expansão da economia estadual. Nesse sentido, parece improvável que a agropecuária - com baixa agregação de

${ }^{10}$ Aqui se destacam principalmente os Tigres Asiáticos (Coréia do Sul, Malásia, Cingapura, e Taiwan). 
valor nas suas atividades (baixo grau inovativo) - isoladamente possa engendrar padrões de crescimento sustentáveis.

Essa mudança estrutural reversa está na contramão da experiência observada em países com crescimento persistente, como, por exemplo, Coreia do Sul. Nesses países existe uma contribuição crescente dos setores industrial (vinculados a atividades de inovação) e de serviços high tech para o crescimento do produto agregado (RADA; TAYLOR, 2006).

\subsection{Fontes do crescimento estadual e a relação emprego-população}

A análise de decomposições empregada nesta seção permite averiguar as fontes (ou efeitos indutores) do crescimento da produtividade do trabalho estadual. Essas fontes são de dois tipos: intrassetorial (ganhos de produtividade) e realocação. O efeito intrassetorial é uma média das taxas de crescimento da produtividade setorial ponderada pelo peso do produto setorial no valor adicionado total. Especificamente, pode-se defini-lo como a variação da produtividade resultante da mudança de produtividade interna aos setores.

O segundo efeito, o realocação, será positivo quando existir crescimento do emprego em setores com produtividade acima da média da economia (RONCOLATO; KUCERA, 2013), ou em setores de baixa produtividade em que o emprego diminua. Nesse caso, a transferência de trabalhadores da agricultura - um setor de baixa produtividade em alguns países -, para a indústria, detentora de alta produtividade, engendrará um crescimento na produtividade média da região.

O gráfico 3 destaca os resultados das decomposições da produtividade setorial entre 1995 e 2002. Nele, pode-se observar a magnitude dos efeitos indutores do crescimento. Por exemplo, verificamos que os três setores exibiram um efeito intrassetorial (ganhos de produtividade) negativo no período. Isso implica crescimento positivo do emprego. A agropecuária foi a única atividade a apresentar um desempenho líquido positivo. 


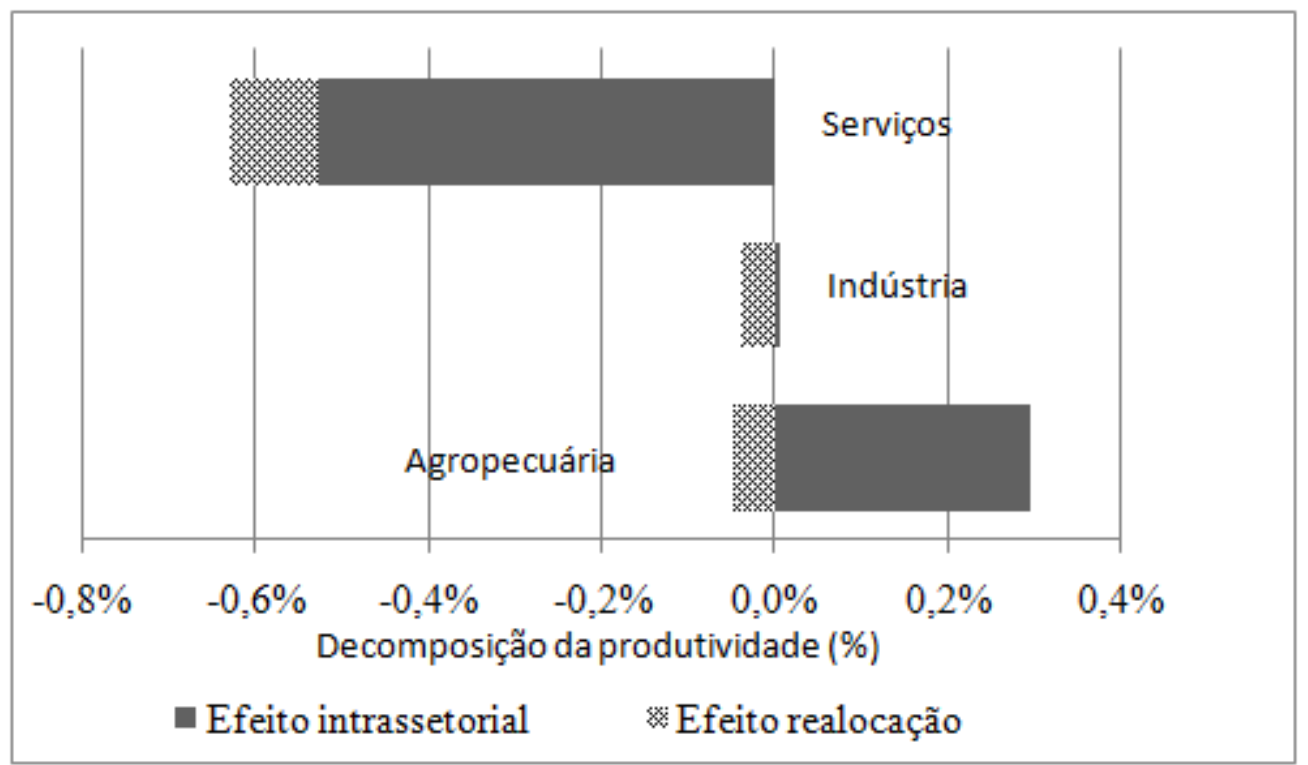

Gráfico 3: Decomposição da produtividade para o Rio Grande do Sul, 1995-2002 Fonte: Elaboração própria.

Duas conclusões podem ser derivadas do Gráfico 3. Primeiro, o setor de serviços exibiu um efeito intrassetorial (em módulo) superior ao efeito positivo da agropecuária. Isto acarretou a determinação de um impacto negativo na produtividade agregada.

Segundo, constata-se que o efeito intrassetorial foi superior ao efeito realocação na explicação dos movimentos da produtividade, confirmando as hipóteses kaldorianas sobre o papel dos fatores internos aos setores (learning by doing, economias externas, e economias de escala dinâmica) como determinantes do desempenho econômico das regiões.

O Gráfico 4 revela os resultados da decomposição da produtividade para o período 2003-2010. Neste período, os serviços e a indústria apresentaram efeitos intrassetoriais negativos que determinaram os resultados da produtividade desses setores. Em contraste, o setor agropecuário exibiu efeitos intrassetorial e realocação positivos, indicando que o setor não contribui de forma decisiva para a geração de empregos. Seu impacto na taxa de crescimento da produtividade, no entanto, foi fundamental para evitar uma retração ainda maior da produtividade total. 


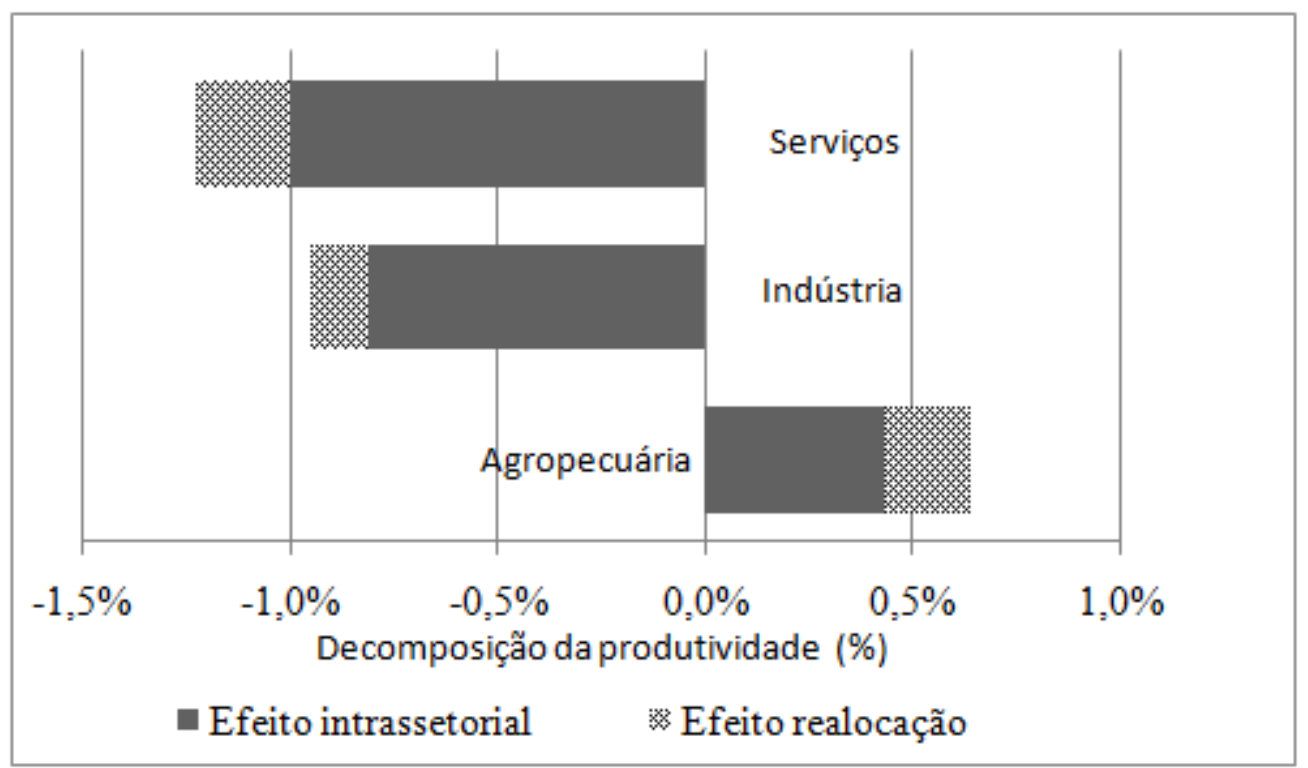

Gráfico 4: Decomposição da produtividade para o Rio Grande do Sul, 2003-2010 Fonte: Elaboração própria.

O Gráfico 5 apresenta as mudanças setoriais na parcela emprego/população do RS entre 2003 e 2010. A relação emprego-população crescerá quando o crescimento do produto per capita exceder a taxa de crescimento da produtividade.

De acordo com as cifras dispostas no Gráfico 5, duas conclusões merecem destaque. Primeiro, os resultados positivos para os serviços e a indústria indicam que os setores contribuíram para a geração de empregos. Como as suas contribuições para o crescimento da produtividade foram negativas, existe um indicativo de que o emprego gerado nesses setores sejam de baixa produtividade ou informal. Segundo, o setor agropecuário exibiu um impacto positivo na geração de emprego (embora pequeno) e crescimento da produtividade, sendo um setor chave da economia gaúcha.

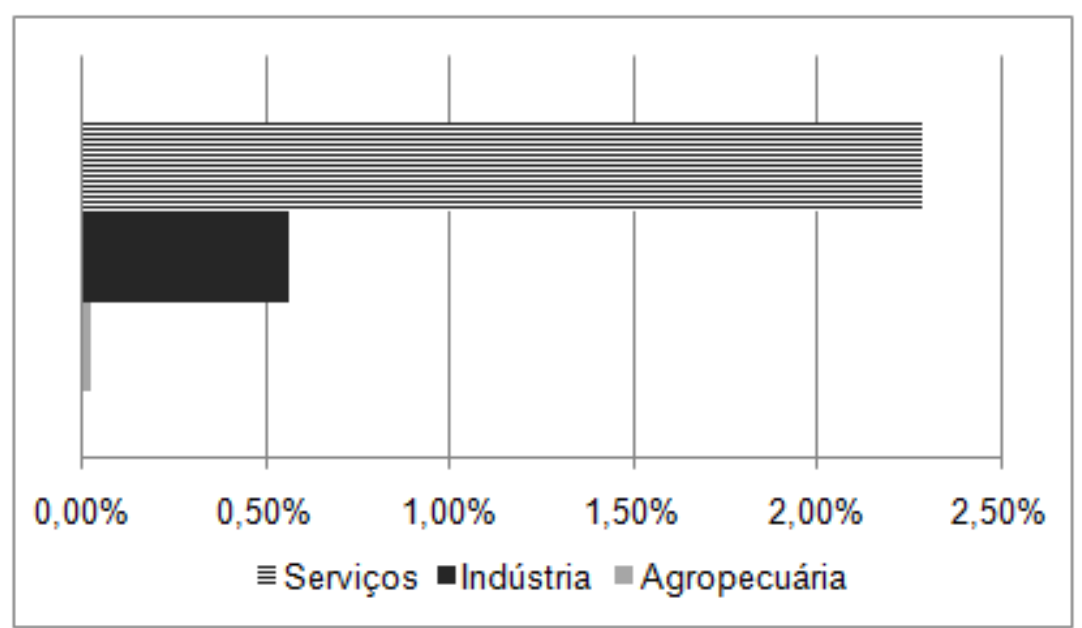

Gráfico 5: Mudanças setoriais na parcela emprego/população do RS, 2003-2010 Fonte: Elaboração própria. 


\subsection{Mudança estrutural dos subsetores da indústria e resumo}

Procedendo-se a uma análise detalhada do setor industrial, ${ }^{11}$ observa-se uma mudança estrutural moderada em direção à atividade manufatureira. Especificamente, a atividade de construção civil e indústria extrativa mineral tiveram suas cifras de participação média reduzidas, ao passo que a indústria de transformação aumentou sua participação de 85,62\% para 85,78\% ao longo dos dois períodos (1995-2002 e 2003-2010).

O Gráfico 6 apresenta a decomposição da taxa de crescimento do produto industrial. Os resultados revelam o aumento em termos relativos da participação da construção civil no crescimento da indústria. A manufatura (indústria de transformação) também contribui para esse crescimento, no entanto, há uma perda de espaço relativo na determinação do produto da indústria. Isso valida as interpretações pessimistas quanto ao processo de desarticulação do setor manufatureiro gaúcho.

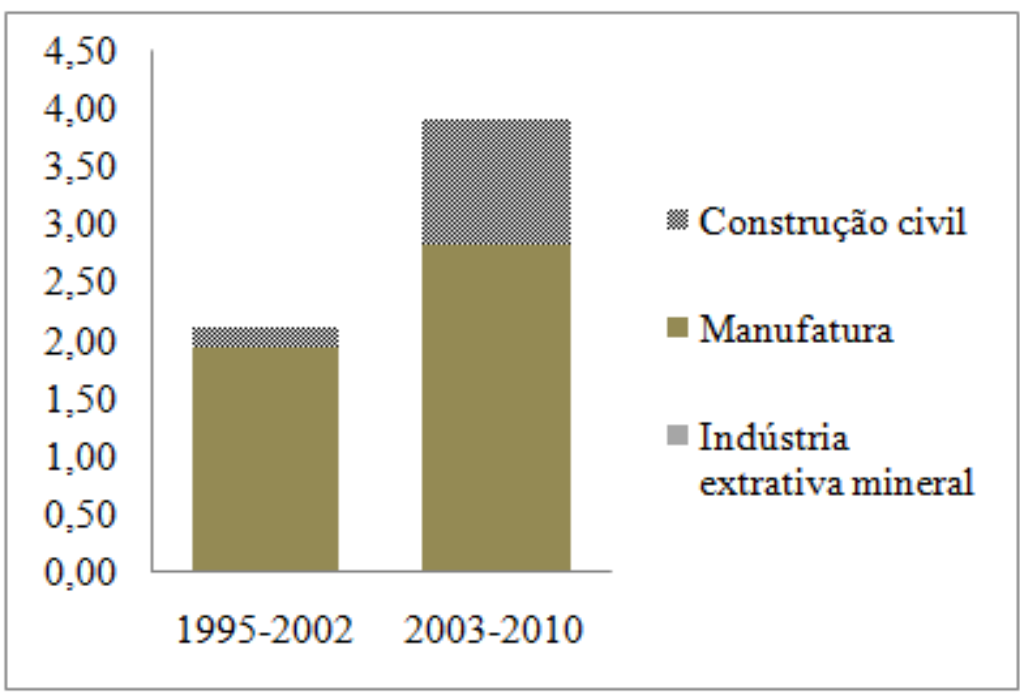

Gráfico 6: Contribuição setorial para o crescimento do produto industrial gaúcho (\%) Fonte: Elaboração própria.

Por fim, o Gráfico 7 mostra a contribuição das atividades para o crescimento da produtividade industrial no período 1995-2010. Constata-se que a performance da indústria de transformação determinou o resultado negativo do setor industrial. A manufatura apresentou uma taxa de crescimento média de aproximadamente $-0,4 \%$ no período 1995-2003. No segundo período, os resultados são ainda piores, exibindo uma taxa média de aproximadamente $-2,3 \%$ ao ano. Essa tendência negativa deve ser revertida a fim de estimular o crescimento da produtividade industrial e da economia. Bender Filho (2013) e Torres at al.

11 Devido à limitação dos dados, neste estudo considera-se o setor industrial como sendo composto pelos seguintes setores: indústria extrativa mineral, indústria de transformação e construção. Nesse sentido, se exclui o setor de abastecimento de água e geração de energia elétrica devido à indisponibilidade de dados. 
(2013), encontraram uma queda similar na contribuição relativa da manufatura, sugerindo a valorização da taxa de câmbio como uma possível causa.

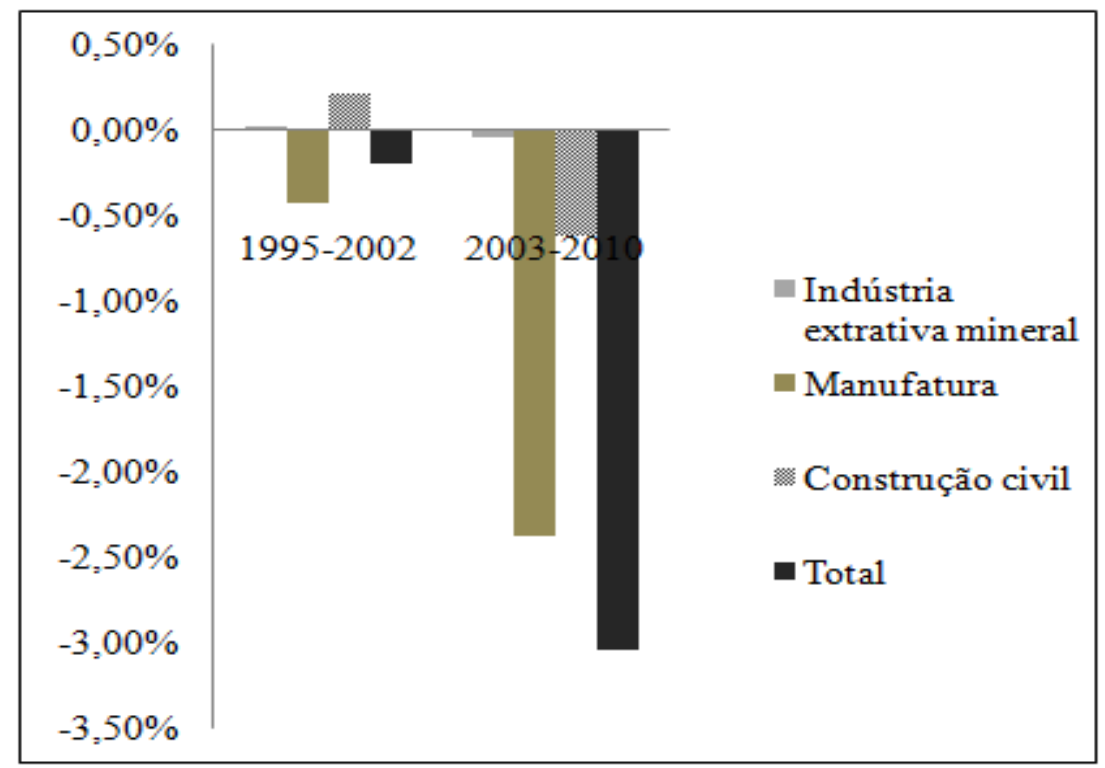

Gráfico 7: Crescimento da Produtividade do trabalho industrial e decomposições setoriais Fonte: Elaboração própria.

Em linhas gerais, pode-se concluir que a mudança estrutural tem afetado negativamente o desempenho da economia nos períodos analisados. $\mathrm{O}$ aprofundamente dessa tendência de mudança estrutural em direção aos serviços (de baixa produtividade) pode ameaçar o processo de crescimento sustentável da economia. Ademais, a manufatura diminuiu sua contribuição relativa na determinação do crescimento da indústria, bem como seu impacto na produtividade industrial entre 2003 e 2010 também colaborarou para a desarticulação do setor industrial. Essa tendência pôde ser destacada de forma mais clara utilizando-se o enfoque dinâmico do método de decomposições.

\section{Conclusão}

O presente estudo empregou a técnica de decomposição a fim de avaliar a contribuição setorial, a direção da mudança estrutural e as fontes para o crescimento da economia gaúcha. As estatísticas da economia gaúcha para o período 1995-2010 foram utilizadas para verificar quais seriam os structural drivers do processo de crescimento estadual.

A performance pífia da indústria tem contribuído negativamente para o desempenho da economia gaúcha no período. As políticas do governo visando ao estímulo do setor industrial foram insuficientes para estimular o setor e o nível de atividade da economia. 
Certamente, o câmbio apreciado foi um fator decisivo para a fraca performance industrial. Nesse sentido, a economia gaúcha parece estar sofrendo um processo de mudança estrutural reversa, em direção a atividades com baixa produtividade. A performance positiva da agropecuária, setor com elevada produtividade, foi insuficiente para evitar os resultados agregados negativos. A combinação de um resultado negativo da indústria com um modelo gerador de crescimento nos serviços de baixa produtividade impede uma expansão vigorosa da economia. O crescimento da produtividade industrial acompanhado de uma demanda efetiva robusta são cruciais para o desenvolvimento da região.

Nesse contexto, verificamos que o efeito intrassetorial foi determinante na explicação do desempenho dos setores nos dois períodos. Em outras palavras, fatores internos aos setores como learning by doing, economias externas, e economias de escala dinâmica são cruciais na determinação do crescimento econômico. Esses fatores são mais importantes do que deslocamentos intersetoriais de mão de obra na explicação do crescimento da produtividade. Isto valida a tese Kaldoriana de que o crescimento se dá via o crescimento de setores com economias de escala dinâmica.

Assim, constata-se que o padrão de mudança estrutural não tem estimulado um crescimento persistente da economia estadual no período 1995-2010. À medida que os serviços ganham importância, é provável uma trajetória de crescimento stop and go, intercalando períodos de aumento do nível de atividade econômica com momentos de estagnação. Nessa medida, o padrão gaúcho de mudança estrutural vai de encontro à evidência internacional de regiões que apresentaram um crescimento sustentável.

Quando comparamos os dois períodos (1995-2002 e 2003-2010), observamos que os resultados negativos da indústria e dos serviços culminaram na queda acentuada da produtividade do trabalho da economia gaúcha no segundo período. Há um aprofundamento das tendências observadas no primeiro período, sendo as mudanças de rumo da política econômica nacional (baseada na distribuição de renda e expansão do crédito a partir de meados de 2003) insuficientes para reverter o processo de deterioração da indústria estadual.

\section{Referências}

BÊRNI, D.; LAUTERT, V. Mesoeconomia: lições de contabilidade social. Porto Alegre: Bookman, 2011. $661 \mathrm{p}$.

BÊRNI, D. Mudanças no padrão de uso da mão-de-obra no Brasil entre 1949 e 2010. Revista Nova Economia, v. 16, n. 1, p. 139-172, 2006. crossrefhttp://dx.doi.org/10.1590/s0103$\underline{63512006000100004}$ 
BENDER FILHO, R. O risco de desindustrialização da economia gaúcha: uma análise do período de 1991 a 2010. Revista Indicadores Econômicos FEE, v. 41, n. 2, p. 67-76, 2013.

CHANG, H.J. Manufacturing: statements, opening statements and manufacturing:statements,rebuttal statements. The Economist, 2011. Disponível em < http://www.economist.com/debate/days/view/714>. Acesso em 20 de março de 2014.

DASGUPTA, S.; SINGH, A. Will services be the new engine of Indian economic growth? Development and Change, v. 36, n. 6, p. 1035-57, 2005. crossref http://dx.doi.org/10.1111/j.0012-155X.2005.00449.x

DIVISIA, F. l'indice monétaire et la théorie de la monnaie. Revue d'Economie Politique, v. 39, p. 980-1008, 1926.

FEIJÓ,C., CARVALHO, P. Uma interpretação sobre a evolução da produtividade industrial no Brasil nos anos 90 e as "Leis de Kaldor". Revista Nova Economia, v. 12, n. 2, p. 57-78, 2002.

FOLEY, D. K.; MICHL, T. R. (1999). Growth and distribution. Cambridge: Harvard University Press, 1999. 355 p.

FUNDAÇÃO DE ECONOMIA E ESTATÍSTICA (FEE/RS). 1995- 2010. Estatisticas econômicas. Governo do Rio Grande do Sul. Disponível em 〈http://www.fee.rs.gov.br〉. Acesso em 10 de fevereiro de 2014.

HARRIS, J.R.; TODARO, M.P. Migration, unemployment and development: a two sector analysis. The American Economic Review, v. 60, n. 1, p. 126-42, 1970.

KALDOR, N. Causes of the slow rate of economic growth in the United Kingdon: an inaugural Lecture. Cambridge, Cambridge University Press, 1966.

KALDOR, N. Productivity and growth in manufacturing industry: a reply. Economica, new series, v. 35, n. 140, p. 385-91, 1968.

KALDOR, N. Economic growth and the Verdoorn Law. A comment on Mr. Rowthorn's article. Economic Journal, Cambridge, v. 85, p. 891-96, 1975. crossref http://dx.doi.org/10.2307/2230633

LEWIS, W. A. Economic development with unlimited supplies of labour. Manchester School, v. 28, n. 2, p. 139-91, 1954. crossrefhttp://dx.doi.org/10.1111/j.14679957.1954.tb00021.x

MANGAIN, V. Are the Kaldor-Verdoorn laws applicable in the newly industrializing countries? Review of Development Economics, Oxford, v. 3, n. 3, p. 295-309, 1999. cross refhttp://dx.doi.org/10.1111/1467-9361.00069

MINISTÉRIO DO TRABALHO E EMPREGO. 1995-2010. RAIS. Governo Federal. Disponível em 〈http://www.mte.gov.br〉. Acesso em 10 de fevereiro de 2014. 
OCAMPO, J. A. The quest for dynamic e_ciency: structural dynamics and economicgrowth in developing countries. In: Ocampo, J. A. (org.). Beyond reforms: structural dynamics and macroeconomic vulnerability. Stanford: Stanford University Press, 2005. crossref http://dx.doi.org/10.1596/978-0-8213-5819-7

OCAMPO, J.A.; C. Rada; L. Taylor. Growth and policy in developing countries: a structuralist approach. New York: Columbia University Press, 2009. 178 p.

OLIVEIRA, F. H. P. Crescimento econômico, retornos crescentes de escala e difusão tecnológica - o caso brasileiro. 2002. 153 p. Dissertação (Mestrado em Economia) Faculdade de Ciências Econômicas da UFMG, Belo Horizonte, 2002. Minas Gerais.

PALMA, J.G. Four sources of "de-industrialization"and a new concept of the "Dutch Disease”. In: José Antonio Ocampo, J. A. ,Beyond reforms:stuctural dynamics and macroeconomic vulnerability. Stanford University Press, 2005.

PASINETTI, L. Structural change and economic growth: a theoretical essay on the dynamics of the wealth of nations. Cambridge: Cambridge University Press, 1981. 281 p.

PREBISCH, R. Commercial Policy in the Underdeveloped Countries. The American Economic Review, v. 49, n. 2, p.251-73, 1959.

RADA, C., TAYLOR, L. Developing and transition economies in the late 20th century: diverging growth rates, economic structures, and sources of demand. SCEPA Working Paper 2006-1. New School for Social Research, 2006.

RADA, C. Stagnation or transformation of a dual economy through endogenous productivity growth. Cambridge Journal of Economics, v. 31, n. 5, p. 711-740, 2007. crossref http://dx.doi.org/10.1093/cje/bem004

RADA, C. Formal and informal sectors in China and India. Economic Systems Research, v. 22, n. 2, p. 315-341, 2010. crossrefhttp://dx.doi.org/10.1080/09535314.2010.482924

RONCOLATO, L.; KUCERA, D. Structural drivers of productivity and employment growth: a decomposition analysis for 81 countries. Cambridge Journal of Economics, v. 38, n. 2, p. 399-424, 2013. crossrefhttp://dx.doi.org/10.1093/cje/bet044

ROS, J. Development and the economics of growth. Ann Arbor: The University of Michigan Press, 2000. 429 p.

SOUZA, N. J. Desenvolvimento regional. São Paulo: Editora Atlas, 2009. 198 p.

STAFFORD, B. Deindustrialization in advanced economies. Cambridge Journal of Economics, v. 13, p. 541-54, 1989.

TAYLOR, L. Macro models for developing countries. New York: McGraw-Hill, 1979. 165 p. 
TAYLOR, L. Structuralist macroeconomics: applicable models for the third world. New York, Basic Books, 1983. 234 p.

THIRLWALL, A. P. A plain man's guide to Kaldor's growth laws. Journal of Post Keynesian Economics, New York, v. 5, n. 3, p. 345-358, 1983. crossref http://dx.doi.org/10.1080/01603477.1983.11489375

TORRES, G. P.; PALERMO, P. U.; PORTUGAL, M. S. O desempenho da indústria no Rio Grande do Sul, entre 1996-2007: uma análise comparada através do método Shift-Share.

Revista Indicadores Econômicos FEE, v. 41, n. 1, p. 45-74, 2013.

VON ARNIM, R.; RADA, C. Labour productivity and energy use in a three-sector model: an application to Egypt. Development and Change, v. 42, n.6, p. 1323-48, 2011. crossref http://dx.doi.org/10.1111/j.1467-7660.2011.01741.x

Recebido em 15.05.14

Aprovado em 10.03.15 